\title{
Automatic DTI-based parcellation of the corpus callosum through the watershed transform
}

\author{
Leticia Rittner*, Pedro Ferro Freitas, Simone Appenzeller, Roberto de Alencar Lotufo
}

\begin{abstract}
Introduction: Parcellation of the corpus callosum (CC) in the midsagittal cross-section of the brain is of utmost importance for the study of diffusion properties within this structure. The complexity of this operation comes from the absence of macroscopic anatomical landmarks to help in dividing the CC into different callosal areas. In this paper we propose a completely automatic method for CC parcellation using diffusion tensor imaging (DTI). Methods: A dataset of 15 diffusion MRI volumes from normal subjects was used. For each subject, the midsagital slice was automatically detected based on the Fractional Anisotropy (FA) map. Then, segmentation of the $\mathrm{CC}$ in the midsgital slice was performed using the hierarchical watershed transform over a weighted FA-map. Finally, parcellation of the CC was obtained through the application of the watershed transform from chosen markers. Results: Parcellation results obtained were consistent for fourteen of the fifteen subjects tested. Results were similar to the ones obtained from tractography-based methods. Tractography confirmed that the cortical regions associated with each obtained $\mathrm{CC}$ region were consistent with the literature. Conclusions: A completely automatic DTI-based parcellation method for the CC was designed and presented. It is not based on tractography, which makes it fast and computationally inexpensive. While most of the existing methods for parcellation of the $\mathrm{CC}$ determine an average behavior for the subjects based on population studies, the proposed method reflects the diffusion properties specific for each subject. Parcellation boundaries are found based on the diffusion properties within each individual CC, which makes it more reliable and less affected by differences in size and shape among subjects.
\end{abstract}

Keywords Corpus callosum, Diffusion tensor imaging, Magnetic resonance imaging, Parcellation, Watershed transform.

\section{Introduction}

The corpus callosum (CC) is an important white matter structure interconnecting the two hemispheres of the brain. Several studies have already shown the relation between alterations of the $\mathrm{CC}$ and diseases such as Alzheimer's (Thompson et al., 2003), schizophrenia (Narr et al., 2000, 2002) and dyslexia (von Plessen et al., 2002). Other studies have assessed the relation of the callosal morphology to gender (DeLacoste and Holloway, 1982; Witelson, 1989), handedness (Witelson and Goldsmith, 1991) and age (Johnson et al., 1994).

Due to its size, the CC is usually divided into smaller regions to facilitate the study of specific portions. These divisions are based on differences of function or histological composition, information that is not available through anatomical magnetic resonance imaging (MRI) techniques. The subdivision of the $\mathrm{CC}$ into a given number of regions, also known as its parcellation, is a very important task with several applications, from studies of the shape and area of the CC section (Biegon et al., 1994; Habib et al., 1991; Hampel et al., 1998; Johnson et al., 1994;
Rumsey et al., 1996) to analysis of the properties inside the structure (O'Dwyer et al., 2011; Park et al., 2011; Rosas et al., 2010) and brain connectivity analysis (Dougherty et al., 2005; Wahl et al., 2007).

As there are no visible landmarks to allow the subdivision of the CC section in the midsagittal slice, several geometrical schemes have been proposed to perform this task (Duara et al., 1991; Larsen et al., 1992; Rajapakse et al., 1996). One of the most important geometrical partitioning schemes was proposed by Witelson (1989), based on post mortem connectivity studies performed in non-human primates and humans. The histological study conducted by Aboitiz et al. (1992), which assessed the fiber composition in the brain with a light microscope, revealed a pattern capable of differentiating the obtained regions. Nevertheless, these studies only reflected the average behavior for a given population. When the proposed geometric schemes are used, the CC is divided the same way for all subjects: in the respective fractions of its maximum extent. The 
properties of each subject are not taken into account in the parcellation.

The development of diffusion tensor imaging (DTI) techniques has provided new information that can be useful in the parcellation of the CC. Diffusion tensor imaging (DTI) is a more recent modality of MRI capable of identifying the direction of water diffusion (Basser and Pierpaoli, 1996). Diffusionweighted MRI sequences acquired for different gradient directions are used to determine the mobility of the water molecules, which can be mathematically represented by a tensor. The direction of the greatest diffusion coefficient corresponds to the direction of the fiber bundle, since the diffusivity of water molecules depends on the principal orientation of the fiber tracts within white matter. DTI makes possible the study of the fiber structure in vivo (Basser et al., 1994) and opens new possibilities for connectivity analysis.

The use of the diffusion properties allowed achieving a parcellation result more representative of the $\mathrm{CC}$ organization than conventional geometric subdivision (Oh et al., 2005). The incorporation of tractography into the parcellation study facilitated the differentiation of the CC regions (Hofer and Frahm, 2006; Huang et al., 2005; Park et al., 2008), determining the cortical areas in which the callosal fibers were projected.

Although these studies are not only based on post-mortem or histological analysis and incorporated important information from diffusion-MRI, the resulting division schemes are still based on the average of the studied population. And as in any of the previous geometric schemes, the resulting CC division is based only on its extent, without taking into account the properties embodied in each subject's MRI.

In opposition, the objective of this paper is to present a method for CC parcellation in DTI specific for each subject and that is at the same time consistent for an entire population. The method uses the watershed transform, and the choice of markers for the watershed is based only on the diffusion properties inside the $\mathrm{CC}$. The method does not use tractography and was designed to be automatic, simple, and fast.

\section{Methods}

The method for the parcellation of the CC presented in this paper consists of the automatic segmentation of the $\mathrm{CC}$ in the midsagittal slice of the brain and its parcellation.

\section{CC segmentation in the midsagittal slice}

The automatic segmentation of the $\mathrm{CC}$ in the midsagittal slice, presented in previous work (Freitas et al., 2011) includes the automatic determination of the midsagittal slice of the brain, the weighting of the FA map, the computation of the morphological gradient and the watershed transform segmentation.

\section{Determination of the midsagittal slice of the brain}

The midsagittal slice of the brain is identified by the diffusion properties observed in the interhemispheric fissure: large areas corresponding to the cerebrospinal fluid (CSF), with low FA values and the white matter structures including the $\mathrm{CC}$ with high FA values. Consequently, by computing the average FA for each slice, after discarding values above a certain limit $F A_{\max }$, the midsagittal slice is identified as the slice with lowest average. This precept is true if we do not take into account slices from extremities with small cross-sectional area of the brain. Therefore, slices with cross-sectional area below a certain minimum $A_{\min }$ are not considered as candidates for midsagittal slice.

\section{Weighted FA map}

The fractional anisotropy (FA) map is a scalar map derived from diffusion-MRI that represents the degree of anisotropy of water diffusion in each voxel, i.e., the degree of existence of a preferential diffusion direction. The FA map reflects a distinct diffusion property inside the $\mathrm{CC}$, since its fibers are highly organized and the fractional anisotropy of the corresponding voxels tends to be high.

As diffusion in the $\mathrm{CC}$ occurs mainly in the leftright direction, since this structure connects the two hemispheres of the brain, the FA values are weighted by the projection $e_{I x}$ of the main eigenvector in the left-right direction to define new weighted values $W_{\text {map }}$ :

$W_{\text {map }}=e_{1 x} * F A$

The objective is to use in segmentation as well in parcellation, not only information about the relation between the eigenvalues present in the FA map, but also the main direction of the fiber bundles.

\section{External morphological gradient}

Since the watershed transform is usually performed over a gradient image, the so-called external morphological gradient is calculated to capture the edges of the $\mathrm{CC}$. The external morphological gradient $G_{e}$ of an image $f$ is defined as the difference between the dilated image and the original image $f$ :

$$
G_{e}(f)=f \oplus b-f
$$

where $\oplus$ denotes the dilation operation using a structuring element $b$. In this case, we used the elementary cross as the structuring element. 


\section{Watershed transform}

The watershed transform was introduced by Digabel and Lantuéjoul (1978) and later used by Beucher and Lantuéjoul (1979) for contour detection. These introductory works described it as an image segmentation method based on a gradient image, where the gray levels are altitudes forming a surface with catchment basins submitted to a flooding process. When two basins touch, a watershed line is raised.

Over the years, several algorithms of watershed have been proposed, according to different formal definitions and applying different strategies. Körbes and Lotufo (2009) showed that most of the algorithms could be described by the two basic graph exploring methods: breadth-first and depth-first. The first watershed algorithm based on a breadth-first method was given by Beucher and Meyer (1992), using a max-cost function implemented through a hierarchical queue. This approach was later shown to be a special case of Dijkstra's generalized algorithm for shortest-path forests called Image Foresting Transform (IFT) using a cost function that is the maximum weight of the edges on the path (Falcão et al., 2004; Lotufo and Falcão, 2000).

Under the image foresting transform (IFT) framework, an image is described as a weighted graph $G=(V, A, w)$ consisting of a set $V$ of nodes representing the image pixels, a set $A$ of arcs weighted by $w$, a function from $A$ to some nonnegative scalar domain. In this context, the watershed transform (IFT-WT) creates a shortest-path forest (SPF), and can be seen as a graph optimization problem. All incident arcs to node $v$ have the same weight, given by the morphological gradient of image $I$ at pixel $v$, $w(u, v)=G(v)$. The IFT-WT assumes that the pathcost function $f_{\max }$ is used:

$$
f_{\max }\left(v_{1}, v_{2}, \ldots, v_{n}\right)=\max \left\{G\left(v_{1}\right), G\left(v_{2}\right), \ldots, G\left(v_{n}\right)\right\}
$$

In other words, the cost function used in the IFT-WT is the maximum weight of the edges on the path and the forest is a minimization of a maximum function. In order to segment objects in an image using the IFT-WT, at least one pixel per object is selected to work as a seed. The IFT-WT returns a SPF where each object is represented by a set of trees rooted at seeds.

In classical watershed, all local minima of the image are used as markers (seeds) for the watershed transform, and the result usually presents oversegmentation, which may not be appropriate for some applications. The local minima can be filtered using some criteria to obtain a more relevant result. This approach is called watershed from markers (Grimaud,
1992). One way to decrease the number of regions is to use the so-called watershed from markers.

In this work, a hierarchical approach is considered in order to properly choose the markers and retain the most significant regions of the image. The local minima with the highest contrast extinction values are used as markers. The contrast extinction value of a regional minimum, also known as the height extinction value, is defined as the minimal climb required for a path starting from a regional minimum to reach another one with strictly lower altitude, the climb being the difference in altitude between the highest point in path and the regional minimum under study (Beucher and Meyer, 1992).

The number $n$ of markers in the watershed transform and, consequently, the number $n$ of regions to be segmented is set to 50. Finally, the obtained regions are grouped to achieve the final segmentation, according to the weighted FA average of each region: all regions within the $\mathrm{CC}$ present a high average, in contrast to the regions outside the CC. A threshold $T=0.2$ of the weighted FA average computed for each region is used to classify the regions that form the CC. The sensibility of the proposed method to the variation of both parameters ( $n$ and $T$ ) were accessed and discussed in previous work (Freitas et al., 2011).

\section{CC parcellation}

The section of the $\mathrm{CC}$ in the midsagittal slice is used to determine the region of interest for the parcellation. The parcellation is done using the watershed transform from markers, based on same weighted FA map and using also the external morphological gradient, this time calculated only for the voxels inside the CC.

\section{Weighted FA map}

By using in the parcellation the same weighted FA map presented in Equation 1, not only the information about the relation between the eigenvalues is used (FA map), but also the main direction of the fiber bundles is taken into account (weighting factor). The weighting in the parcellation is crucial and is what allows the watershed to distinguish $\mathrm{CC}$ portions connected to different cortex regions.

\section{External morphological gradient}

The external morphological gradient is again used, this time in order to obtain the internal divisions of the CC. As the weighted FA map for the voxels of the $\mathrm{CC}$ are significantly higher than the voxels of the background, the result for the voxels for the $\mathrm{CC}$ is not affected by the voxels of the background. It would not be desirable that the borders obtained inside the $\mathrm{CC}$ were influenced by the voxels of the background. 


\section{Watershed transform}

The watershed transform is again calculated over the external morphological gradient, this time to obtain the division of the $\mathrm{CC}$, based only on the diffusion properties within the structure. The main difference between the watershed used in the segmentation step and parcellation is that the position of the markers is determined not by the dynamics of the image, but by points along the centerline of the $\mathrm{CC}$, to adapt to variations in size and shape of the $\mathrm{CC}$. The construction of the centerline of the $\mathrm{CC}$ follows the method presented by Park et al. (2011), with minor alterations.

First, the pixels corresponding to the border of the $\mathrm{CC}$ are determined from its section in the midsagittal slice (Figure 1a). These pixels are then divided into superior and inferior borders from the extreme points (Figure 1b). These points are determined automatically according to the shape of the CC: in the posterior and the anterior regions the extreme points are the most inferior ones (in red). A curve representing each border is calculated using a cubic spline (Figure 1b), with 200 equally spaced points. Pairs of corresponding points along the curves are connected and the midpoint of each segment is chosen to construct the centerline of the CC (Figure 1c).

The $25^{\text {th }}, 80^{\text {th }}, 115^{\text {th }}, 140^{\text {th }}$ and $170^{\text {th }}$ points along the centerline of the $\mathrm{CC}$ (counting from the anterior extremity) were chosen to originate the markers for the watershed transform based on a study presented in the next section. The arrows in Figure 2a indicate the chosen points, each point representing a region of the CC. The pixels in which these points are located are assigned as markers for the watershed transform (Figure 2b). The parcellation is then performed using the watershed transform from these markers (Figure 2c). It is important to emphasize that, despite using the same anatomically roughly homologous points for all subjects, the boundary between two neighboring regions is still determined by the variation in the diffusion properties along the $\mathrm{CC}$ of each subject.

\section{Method rationale}

The method proposed in this paper performs the division of the $\mathrm{CC}$ with a particular choice of markers for the watershed transform. The position of the markers is determined from points chosen along the centerline of the $\mathrm{CC}$. The rationale for the choice and positioning of the watershed markers is summarized in this section.

Initial experiments were performed to evaluate the parcellation of the $\mathrm{CC}$ using the watershed transform. The hierarchical approach of the watershed transform was used to divide the $\mathrm{CC}$ automatically using only the diffusion information within the structure. The markers chosen for the watershed transform were the local minima with highest extinction values. This approach was used to obtain the most important regions in the interior of the $\mathrm{CC}$, where there was no control for the position of the markers. The parcellation was performed for all fifteen subjects using the same number of regions $\left(n_{p}=5\right)$.

For eight of the fifteen subjects tested (Figures 3a-h), the parcellation presented a visual pattern: one region in the anterior end, one region in the posterior end, and the central portion divided into three regions: one larger in the anterior portion and
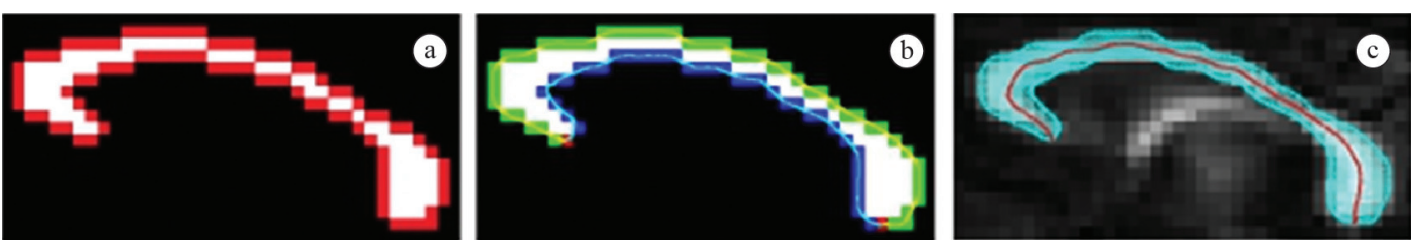

Figure 1. Steps of the construction of the CC centerline: (a) the determination of the voxels corresponding to the border of the CC, (b) the division of the border into superior (green) and inferior (blue), with the calculation of the respective curves (yellow and cyan), and (c) the connection of the corresponding points along the curves (cyan), with the selection of the midpoint of each segment to construct the center line of the CC (red)(Color figure online).
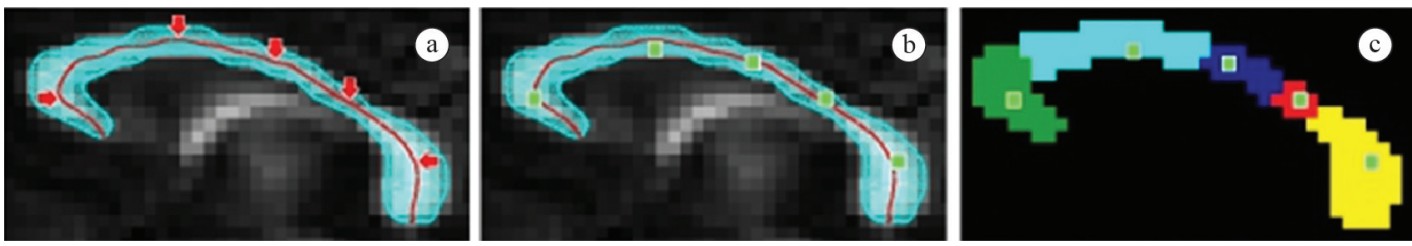

Figure 2. Illustration of the markers for the watershed transform for a single subject: (a) points indicated along the centerline, (b) pixels and (c) result of the watershed transform. 
two smaller in the posterior portion. For the remaining subjects (Figures 3i-o), it was not possible to establish a parcellation pattern similar to the one previously observed (Figures 3a-h). Some problems, like the lack of a small region anterior end (Figures $3 i-j$ and $3 n$ ) and the region in the posterior end split horizontally (Figure $3 \mathrm{k}$ and $3 \mathrm{~m}$ ), led to an undesirable parcellation for some subjects.

After the initial experiments with the parcellation, an analysis comparing the diffusion properties inside the $\mathrm{CC}$ and the preliminary parcellation results was done. The analysis was carried out to understand how the information in the FA map may help the parcellation and to determine what led to different results for some subjects. The objective of the study was to establish a method for the placement of watershed markers. The study followed the method presented by Park et al. (2011), with minor alterations to make it simpler, and looked for a relation between the CC internal characteristics and the parcellation results obtained by the initial approach. Differently from the method used in Park et al. (2011), in our method the bottom line points were determined exactly as the top line points (200 equidistant surface points). Then the FA was mapped onto the 200 midline points not by searching for the maximum value along the line connecting the top-bottom correspondence points, but by using the FA value from the voxel closest to the midline.

The construction of the centerline of the CC followed the same steps previously described and depicted in Figure 1c. Four different scalar maps obtained from DTI were used to assess the diffusion characteristics inside the CC: axial diffusivity, fractional anisotropy (FA), mean diffusivity (MD) and radial diffusivity. These maps were evaluated for all 15 subjects in the two hundred points along the centerline of the $\mathrm{CC}$. The mean value between all subjects for each point, and for each scalar map, was plotted in red in Figure 4. The curves above and below the mean value represent the addition and subtraction of the standard deviation calculated between all subjects for each point.

The variation of the curves between subjects, represented by the standard deviation in each point, is small, and each curve presents clearly distinct regions that can be related to the parcellation results. The fractional anisotropy (Figure 4b) presents two peaks at the extremities of the $\mathrm{CC}$, and a deep valley next to the peak in the posterior region. On the other hand, mean diffusivity (Figure 4c) and radial diffusivity (Figure 4d) present a complementary behavior. Axial diffusivity (Figure 4 a) is the only property among those studied that does not present significant variation.

After plotting different diffusion properties for the points along the centerline of the $\mathrm{CC}$, the study focused on the FA map, since it was used in the parcellation and the other diffusion properties analyzed did not add any new information that might explain the results or help in this task. The objective was to determine in which region of the parcellation the points along the centerline were located, and thus determine
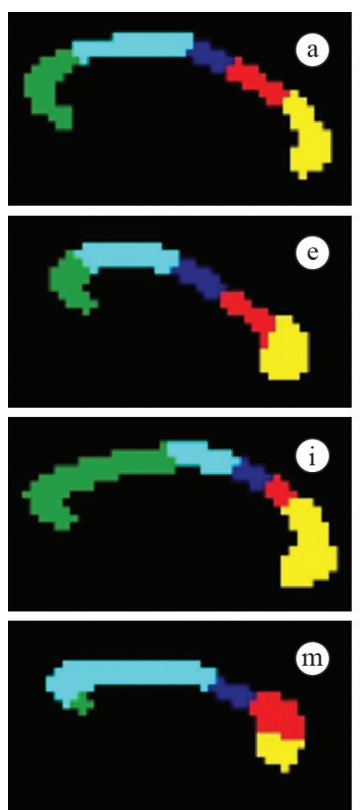
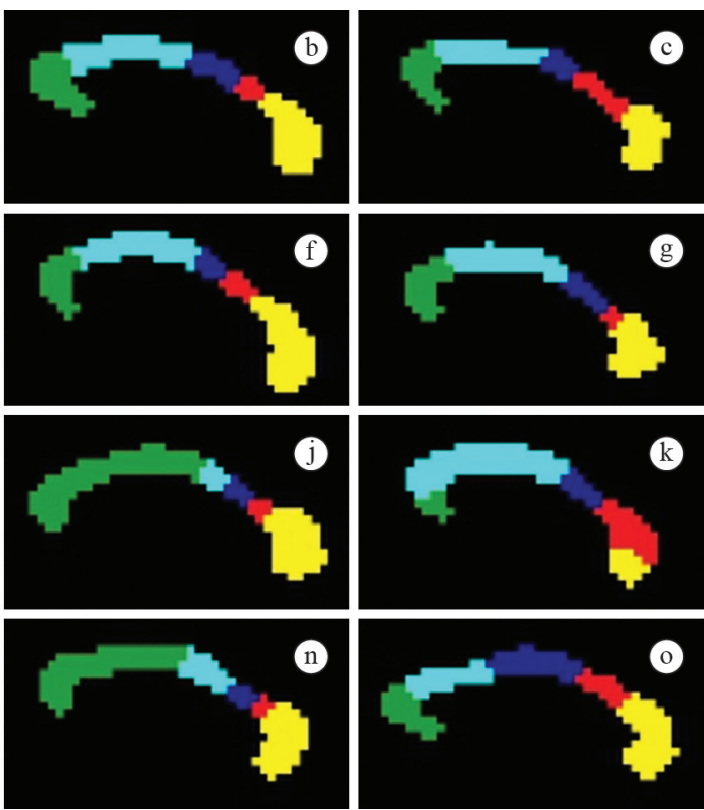
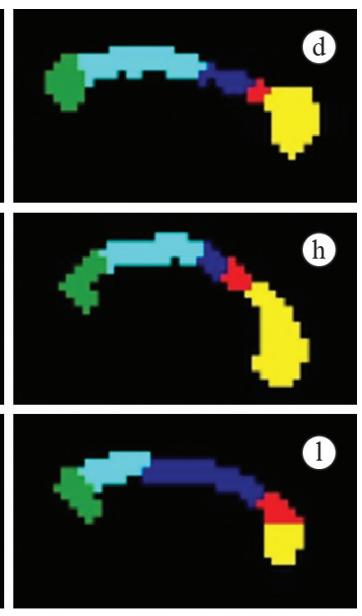

Figure 3. Result of the parcellation in five regions with the hierarchical approach. (a)-(h): for the first eight subjects the presented division was visually similar; (i)-(o): for the remaining subjects the division did not followed the established pattern. 

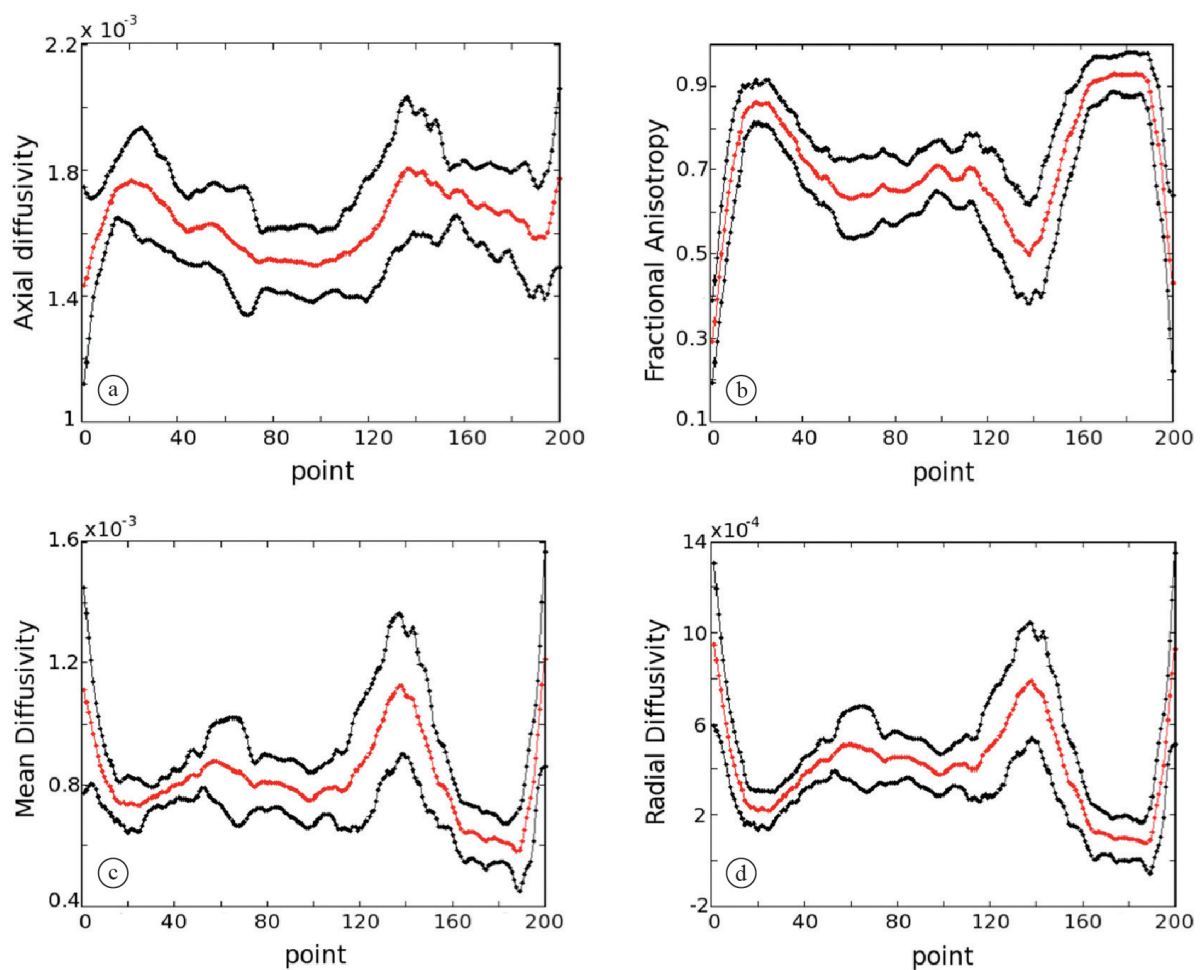

Figure 4. Mean value profile plots of diffusion indices for the fifteen subjects: (a) axial diffusivity, (b) fractional anisotropy, (c) mean diffusivity, (d) radial diffusivity. The $\mathrm{x}$-axis represents the 200 points along the centerline of the CC.

the point corresponding to each boundary between neighboring regions. Only the result for the subjects whose parcellation followed the established pattern (Figures 3a-h) were taken into account.

The points along the centerline that correspond to the boundaries between neighboring regions were calculated for all the chosen subjects. For each boundary, the points obtained for all the subjects were plotted over the curve of the FA map (Figure 5a). A blue line represents the minimum point for a given division among all subjects, while a green line represents the maximum point. The range between the minimum and the maximum points, in cyan, represents a particular boundary inside the $\mathrm{CC}$.

The process was repeated for other main existing theoretical schemes (Witelson's scheme, Aboitiz's scheme, Hofer and Frahm's scheme), in order to observe the diffusion properties together with the location of the boundaries. Among the analyzed schemes, the results from Hofer and Frahm (2006) came closest to the method proposed here.

The changes in the FA map are directly related to the position of the division between the regions obtained from the parcellation by the proposed method (Figure 5a) and by the method proposed by Hofer and Frahm (Figure 5b). The divisions were always placed in the area where the diffusion characteristics, in this case the FA, change significantly. The analysis inside the $\mathrm{CC}$ showed that there was information in the FA map to allow its parcellation, even influenced by the position of the watershed markers. The close relation between the diffusion properties and the boundaries between the regions in the initial experiments presented the most important improvement opportunities explored in the proposed parcellation method.

For each of the five regions in the parcellation scheme one point along the centerline of the CC was chosen to represent it and to place one marker for the watershed transform (arrows in Figure 6). Regions I, IV and V were represented by the points of minimum gradient within each region: $25^{\text {th }}, 140^{\text {th }}$ and $170^{\text {th }}$ point, respectively. Regions II and III present no point of minimum gradient, therefore their central point $\left(80^{\text {th }}\right.$ and $\left.115^{\text {th }}\right)$ were chosen for the last two watershed markers. While the boundary between two neighboring regions was still determined by the variation in the diffusion properties, the positioning of the watershed markers is no longer determined by the hierarchy of the local minima, but by the diffusion properties in the $\mathrm{CC}$, being less affected by differences in shape and size of the CC. It is also important to point out that, since these markers are only starting points used by the watershed to find borders between regions based on the weighted FA 

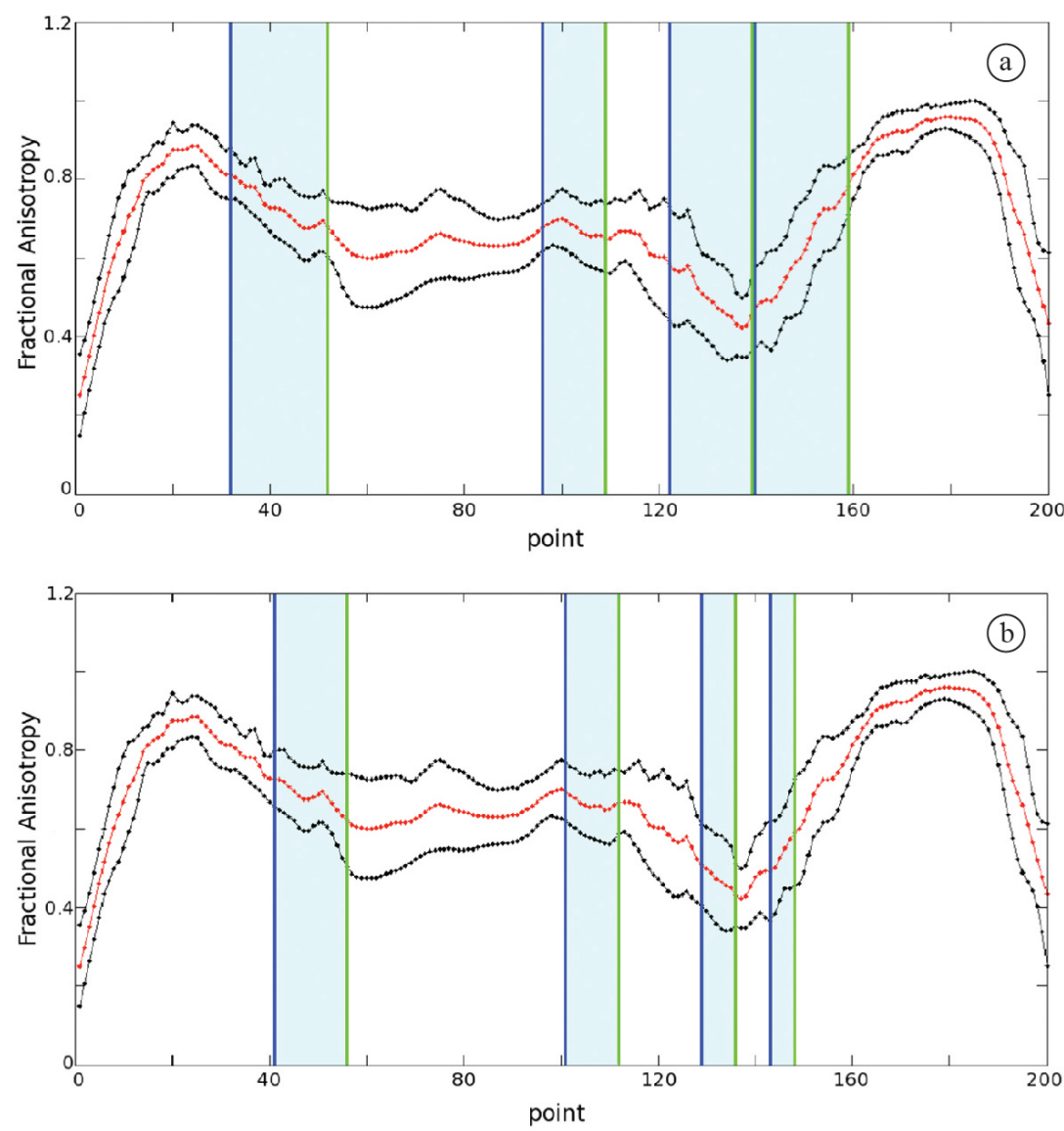

Figure 5. Location of the parcellation boundaries relative to mean value profile plots of the fractional anisotropy for the fifteen subjects: (a) our method, (b) Hofer and Frahm method (2006). The x-axis represents the 200 points along the centerline of the CC. The range (cyan) between the minimum point (blue) and the maximum point (green) referent to each division is overlaid to the original plot (Color figure online).

map, small variations in these points do not imply in changes in parcellation results.

\section{Results}

\section{Acquisition}

The diffusion data used in the experiments were acquired on a Siemens 3T Trio MR scanner using an 8-channel phased array head coil: diffusion images with $N=30$ diffusion encoding directions with $b=1000 \mathrm{~s} / \mathrm{mm}^{2}, 2.0 \mathrm{~mm}$ isotropic voxel size, 63 slices, $T E=95 \mathrm{~ms}, T R=8700 \mathrm{~ms}$. The test set consists of 15 volumes, acquired for 15 different normal subjects.

\section{Parcellation}

Parcellation of the $\mathrm{CC}$ into five regions was performed for all fifteen subjects, to evaluate the performance of the proposed approach, with the markers for the watershed transform positioned at the $25^{\text {th }}, 80^{\text {th }}, 115^{\text {th }}$, $140^{\text {th }}$ and $170^{\text {th }}$ points along the centerline of the CC. For the eight subjects who had the parcellation in the preliminary experiments already following the pattern (Figures 3a-h), the result from the proposed method still followed the pattern (Figures 7a-h). The parcellation also followed the pattern for most other subjects (Figures 7i-n). For only one subject (Figure 7o) the parcellation was still different from the pattern, with the second region from the anterior end (in cyan) significantly smaller than in other subjects.

The parcellation result in the initial experiments was visually similar only for eight of the fifteen subjects and became similar for fourteen subjects with the proposed approach, attesting to the importance of the positioning of the markers.

\section{Tractography}

As parcellation should reflect the function of the different regions of the $\mathrm{CC}$, the evaluation of the parcellation results was done through tractography of the callosal fibers.

Fiber tracking was done using the MINC Diffusion Tools (http://www.bic.mni.mcgill.ca/ServicesSoftware/ MINC). It runs with the FACT (Fiber Assignement 
by Continous Tracking) algorithm (Mori et al., 1999), which uses the principal eigenvector direction for tracking. The FA threshold was set to 0.15 and the curvature constraint angle was set to 40 degrees (Hofer and Frahm, 2006). The five regions resulting from parcellation were used as seeds for tractography. Tractography was performed to confirm to which area of the cerebral cortex the fibers originating in the regions resulting from parcellation were connected. For each region of the $\mathrm{CC}$ a particular color was assigned in the tractography result.
Tractography results confirm that the cortical regions associated with each CC subdivision (Figure 8) are consistent with the literature. Most of the fibers in region I (green) of the $\mathrm{CC}$ are connected to the prefrontal cortex. Regions II, III and IV (cyan, blue and red, respectively) map to pre-motor, supplementary motor, motor and sensory cortical areas. The exact border between the fibers coming from different regions cannot be determined, but Figure $8 \mathrm{c}$ shows that the fibers are mapped similarly to Hofer and Frahm (2006). Region V (yellow) is connected to the parietal, occipital, and temporal cortices.

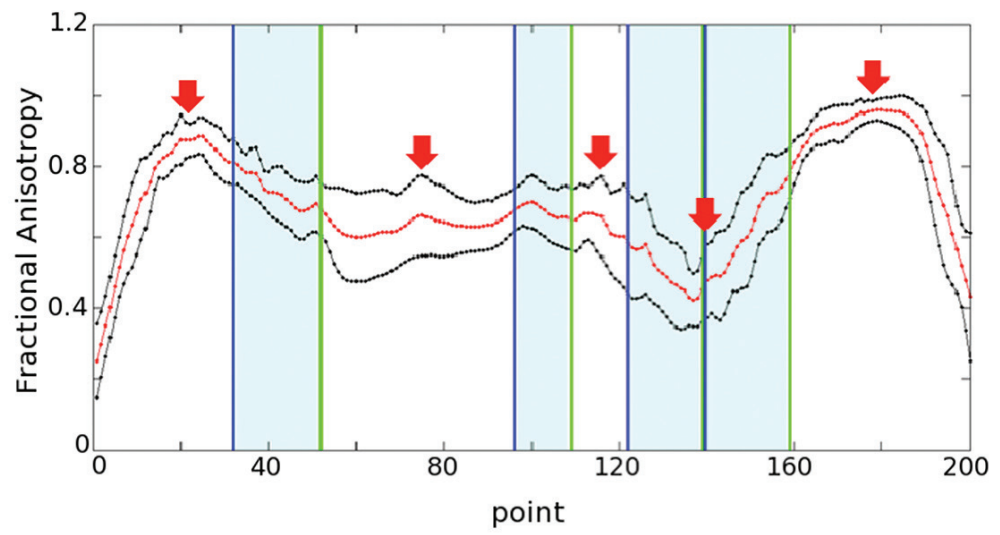

Figure 6. Mean value profile plots of the FA map for the fifteen subjects. The x-axis represents the 200 points along the centerline of the CC. The points which determine the position of the watershed makers are indicated by red arrows. The range (cyan) between the minimum point (blue) and the maximum point (green) referent to each division obtained with the initial approach of the proposed method is overlaid to the original plot.
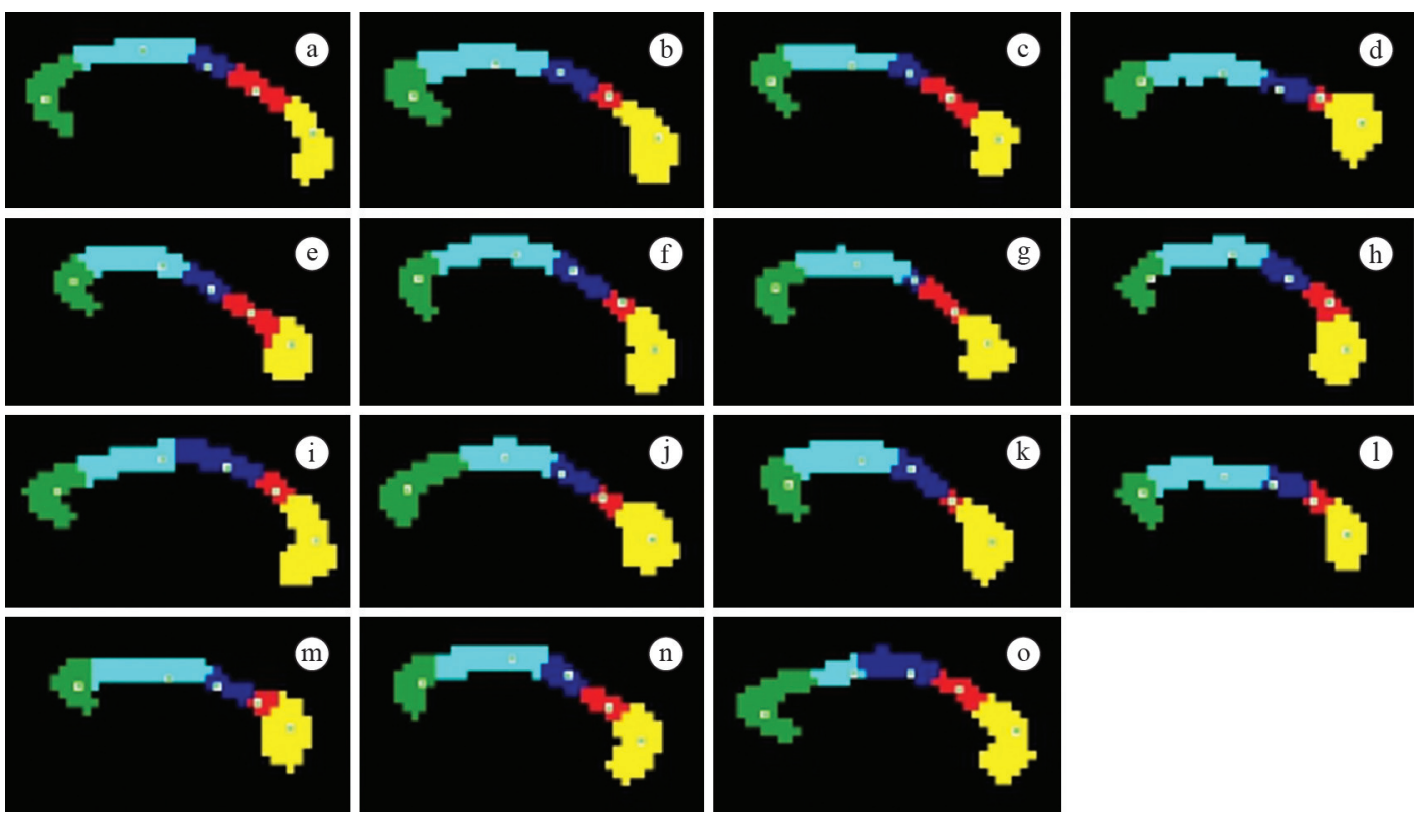

Figure 7. Results of the parcellation in five regions with markers positioning: (a)-(h): for the eight subjects for which the parcellation using the initial hierarchical markers selection followed the pattern; (i)-(o) for the remaining subjects, for which the parcellation using hierarchical markers selection did not follow the pattern. 

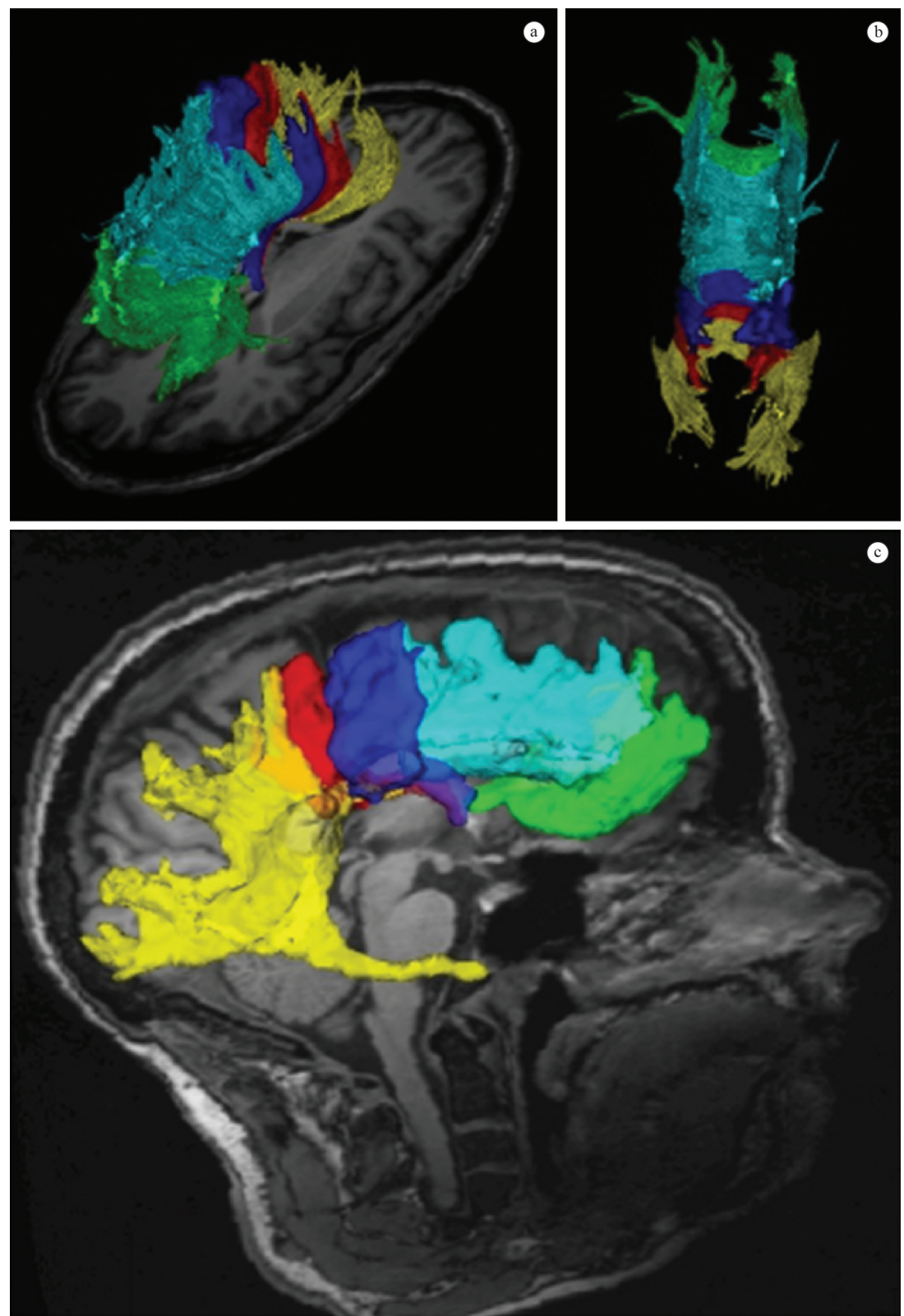

Figure 8. Tractography results for a single subject overlaid in the midsagittal slice of the brain in the T1-weighted MRI.

\section{Discussion}

The proposed method was able to perform the parcellation of the $\mathrm{CC}$ robustly for fourteen of the fifteen subjects of the test set (Figures 7a-n). The placement of the watershed markers based on fractional anisotropy along the center line of the $\mathrm{CC}$ (Figure 6) was essential to deal with the problem faced in the placement of the markers in hierarchical watershed, where the wrong placement of the markers led to incorrect parcellation for some subjects (Figures 3i-o).

In accordance with previous study presented by Hofer and Frahm (2006), the proposed method was able to distinguish among five segments of the $\mathrm{CC}$ based on the connectivity of the callosal fibers. The obtained distribution of the regions was also similar to the distribution observed using the scheme of Hofer and Frahm (2006) (Figure 5). Most of the differences come from the fact that the division made by our method is specific to each subject, while the scheme proposed by Hofer and Frahm divides all subjects the same way.

Witelson (1989) also divided the CC into five regions, but there are significant differences in the anterior tip and the midbody area. While Witelson defined region I as the anterior third, the first region 
we obtained is approximately half this size. On the other hand, region II, which was defined by Witelson as the remaining anterior half, is significantly larger for our method. The differences were possibly caused by shrinkage and deformation of the brain during the post-mortem fixation procedure and the use of non-human primate data

Oh et al. (2005) also use the diffusion properties to cluster small subdivisions inside the $\mathrm{CC}$ into nine regions. Despite the difference in the number of regions between the methods, there is an excellent correspondence in the main divisions obtained. The three regions obtained by $\mathrm{Oh}$ et al. in the anterior end are equivalent to the region obtained in the anterior portion by our method. The same applies to the two regions obtained by $\mathrm{Oh}$ et al. in the posterior end and the posterior region obtained by us. The approximate division of the $\mathrm{CC}$ in half is also present in both approaches. Increasing the number of regions in the watershed transform for our parcellation method leads to an approximation of the results by Oh et al. (2005), even with the markers determined by the hierarchy of local minima. Due to our choice of the markers, it is not possible to differentiate between the dorsal and ventral areas of the CC.

As the other parcellation methods based on DTI (Huang et al., 2005; Park et al., 2008) are not focused on dividing the $\mathrm{CC}$ into distinct regions, the comparison with our proposed method is not possible.

The evaluation of the diffusion properties along the centerline of the CC (Figure 4) was important both to give clues about the improvements to be made in the presented method and to compare with other studies. Diffusion properties, especially fractional anisotropy, can reveal the microstructure of brain white matter such as fiber diameter and density (Chepuri et al., 2002; Oh et al., 2005).

The obtained distribution of fractional anisotropy along the $\mathrm{CC}$ was similar for the entire test set, even with its absolute value varying between subjects, as previously reported (Hofer and Frahm, 2006). Higher fractional anisotropy values were found in the extremities of the CC. Lower FA observed in the posterior midbody regions, which would be connected to the primary motor and sensory cortical areas, were also in accordance with the findings by Hofer and Frahm (2006). The observed FA values are in accordance with structural organization reported by Aboitiz et al. (1992). The concentration of fibers with a relatively small diameter in the anterior and posterior portions of the $\mathrm{CC}$ is reflected in high FA values. Less densely packed fibers with considerably larger diameters, which are concentrated in the posterior midbody, explain the low FA values in these regions.

The four diffusion properties we evaluated inside the $\mathrm{CC}$ in Figure 4 (axial diffusivity, fractional anisotropy, mean diffusivity, and radial diffusivity) presented a very similar behavior to the work presented by Park et al. (2011), even using a completely different test set with non-interpolated data.

In the parcellation using the proposed method, the position of the boundaries between neighboring regions was closely related to the diffusion properties, especially to the FA map (Figure 6). As the determination of the watershed markers is also based on the diffusion properties, the proposed method should work correctly even when used on new datasets, since it is expected that the diffusion properties inside the $\mathrm{CC}$ remain unchanged.

The obtained parcellation for only one subject was different from the common pattern, using not only hierarchical marker selection (Figure 3o) but also markers based on diffusion properties (Figure 7o). Variations in the segmentation of the CC section in the midsagittal slice, as for example larger anterior end than in other subjects, may have affected the placement of the watershed markers and caused the parcellation to fail.

In contrast to most methods presented using DTI (Hofer and Frahm, 2006; Huang et al., 2005; Park et al., 2008), in which tractography was use to construct the parcellation scheme, in this work tractography was used for the validation of the proposed method. The regions obtained in the parcellation were used as seeds to show that the fibers coming from different regions of the $\mathrm{CC}$ are mapped into different areas of the cerebral cortex.

The tractography results presented in this paper are still preliminary, since they were obtained for a single subject, but the fiber tracking has already shown that the subdivisions of the CC correspond mostly to the expected cortex regions. The division between the fiber bundles going to different regions is not so clear as presented by Hofer and Frahm, despite similarity of mapping to the cortical areas (Figure 8). In fact, in some boundaries there are even overlaps. But this is not different from results reported by Hofer and Frahm (2006) and Huang et al. (2005). Tractography is affected by partial volume effects, crossing fibers, low resolution and unsolved issues of the tractography algorithm. What is important to point out is that our parcellation results do not rely on tractography, but used it here only to enrich the discussion of the results.

In summary, we proposed a completely automatic method for the parcellation of the CC based on the diffusion properties within the structure. It uses the watershed transform and includes the determination of the midsagittal slice, the $\mathrm{CC}$ segmentation in this slice and an automatic positioning of watershed markers based on FA along the centerline of CC. 
Since the method performs the parcellation based on the diffusion information within the $\mathrm{CC}$ of each subject, and not based on an average geometry for a studied population, it is more reliable and less affected by differences in size and shape among subjects. Unlike recent studies, the proposed method does not use tractography to map the cortex regions where the callosal fibers are connected, which makes it fast and computationally inexpensive.

The results obtained were consistent for fourteen of the fifteen subjects tested, leading us to conclude that a subject-specific parcellation is feasible.

\section{Acknowledgments}

The authors would like to thank the National Council for Scientific and Technological Development (CNPq), the Coordination for the Improvement of Higher Education Personnel (CAPES) and the São Paulo Research Foundation (FAPESP) for funding this research.

\section{References}

Aboitiz F, Scheibel AB, Fisher RS, Zaidel E. Fiber composition of the human corpus callosum. Brain Research. 1992; 598(12):143-53. http://dx.doi.org/10.1016/0006-8993(92)90178-C

Basser PJ, Pierpaoli C. Microstructural and physiological features of tissues elucidated by quantitative-diffusiontensor MRI. Journal of Magnetic Resonance, Series B. 1996; 111(3):209-19. http://dx.doi.org/10.1006/ jmrb.1996.0086

Basser PJ, Mattiello J, LeBihan D. MR diffusion tensor spectroscopy and imaging. Biophysical Journal. 1994; 66(1):259-67. http://dx.doi.org/10.1016/ S0006-3495(94)80775-1

Beucher S, Lantuéjoul C. Use of watersheds in contour detection. In: International Workshop on Image Processing: Proceedings of the International Workshop on Image Processing: Real-time Edge and Motion Detection/ Estimation; 1979; Rennes, France.

Beucher S, Meyer F. The morphological approach to segmentation: The Watershed Transformation. Mathematical Morphology in Image Processing (CRC Press). 1992. p. $433-81$.

Biegon A, Eberling JL, Richardson BC, Roos MS, Wong ST, Reed BR, Jagust WJ. Human corpus callosum in aging and alzheimer's disease: a magnetic resonance imaging study. Neurobiology of Aging. 1994;15(4):393-7. http:// dx.doi.org/10.1016/0197-4580(94)90070-1

Chepuri NB, Yen YF, Burdette JH, Li H, Moody DM, Maldjian JA. Diffusion anisotropy in the corpus callosum. American journal of Neuroradiology. 2002; 23(5):803-8. PMid:12006281.

DeLacoste-Utamsing C, Holloway R. Sexual dimorphism in the human corpus callosum. Science. 1982; 216(4553):14312. http://dx.doi.org/10.1126/science.7089533
Digabel H, Lantuéjoul C. Iterative algorithms. In: European Symposium Quantitative Analysis of Microstructures in Material Science, Biology and Medicine: Proceedings of the 2nd European Symposium Quantitative Analysis of Microstructures in Material Science, Biology and Medicine; 1978. p. 85-99.

Dougherty RF, Ben-Shachar M, Bammer R, Brewer AA, Wandell BA. Functional organization of human occipitalcallosal fiber tracts. Proceedings of the National Academy of Sciences of the United States of America. 2005; 102(20):73505. PMid:15883384 PMCid:PMC1129102. http://dx.doi. org/10.1073/pnas.0500003102

Duara R, Kushch A, Gross-Glenn K, Barker WW, Jallad B, Pascal S, Loewenstein DA, Sheldon J, Rabin M, Levin B, Lubs H. Neuroanatomic differences between dyslexic and normal readers on magnetic resonance imaging scans. Archives of Neurology. 1991; 48(4):410-6 . PMid:2012516. http://dx.doi.org/10.1001/archneur.1991.00530160078018

Falcão AX, Stolfi J, Lotufo RA. The image foresting transform: theory, algorithms, and applications. IEEE Transactions on Pattern Analysis and Machine Intelligence. 2004; 26(1):19-29. PMid:15382683. http:// dx.doi.org/10.1109/TPAMI.2004.1261076

Freitas P, Rittner L, Appenzeller S, Lotufo RA. Watershedbased segmentation of the midsagittal section of the corpus callosum in diffusion MRI. In: Graphics, Patterns and Images, Conference on: Proceedings of the 24th Conference on Graphics, Patterns and Images; 2011. IEEE Computer Society; 2011. p. 274-80.

Grimaud M. A new measure of contrast: the dynamics. Image Algebra and Morphological Image Processing III. 1992; 1769:292-305. http://dx.doi.org/10.1117/12.60650

Habib M, Gayraud D, Oliva A, Regis J, Salamon G, Khalil R. Effects of handedness and sex on the morphology of the corpus callosum: a study with brain magnetic resonance imaging. Brain and Cognition. 1991;16(1):41-61. http:// dx.doi.org/10.1016/0278-2626(91)90084-L

Hampel H, Teipel SJ, Alexander GE, Horwitz B, Teichberg D, Schapiro MB, Rapoport SI. Corpus callosum atrophy is a possible indicator of region- and cell type-specific neuronal degeneration in Alzheimer disease: a magnetic resonance imaging analysis. Archives of Neurology. 1998; 55(2):193-8. PMid:9482361. http://dx.doi.org/10.1001/archneur.55.2.193

Hofer S, Frahm J. Topography of the human corpus callosum revisited-comprehensive fiber tractography using diffusion tensor magnetic resonance imaging. NeuroImage. 2006; 32(3):989-94. PMid:16854598. http:// dx.doi.org/10.1016/j.neuroimage.2006.05.044

Huang H, Zhang J, Jiang H, Wakana S, Poetscher L, Miller MI, van Zijl PC, Hillis AE, Wytik R, Mori S. DTI tractography based parcellation of white matter: application to the mid-sagittal morphology of corpus callosum. NeuroImage. 2005; 26(1):195-205. PMid:15862219. http:// dx.doi.org/10.1016/j.neuroimage.2005.01.019

Johnson SC, Farnworth T, Pinkston JB, Bigler ED, Blatter DD. Corpus callosum surface area across the human adult life span: Effect of age and gender. Brain Research Bulletin. 1994; 35(4):373-7. http://dx.doi.org/10.1016/03619230(94)90116-3 
Körbes A, Lotufo RA. Analysis of the watershed algorithms based on the Breadth-First and Depth-First exploring methods. In: Computer Graphics and Image Processing, Brazilian Symposium on: Proceedings of the 22th Brazilian Symposium on Computer Graphics and Image Processing; 2009; Rio de Janeiro, Brazil. IEEE Computer Society; 2009. p. 133-40. http://dx.doi.org/10.1109/SIBGRAPI.2009.43

Larsen JP, Höien T, Odegaard H. Magnetic resonance imaging of the corpus callosum in developmental dyslexia. Cognitive Neuropsychology. 1992; 9(2):123-34. http:// dx.doi.org/10.1080/02643299208252055

Lotufo RA, Falcão AX. The ordered queue and the optimality of the watershed approaches. In: Mathematical Morphology and its Applications to Image and Signal Processing: Proceedings of the 5th International Symposium on Mathematical Morphology and its Applications to Image and Signal Processing; 2000. Kluwer Academic Publishers; 2000. v. 18, p. 341-50. http://dx.doi.org/10.1007/0-306-47025-X_37

Mori S, Crain BJ, Chacko VP, van Zijl PCM. Threedimensional tracking of axonal projections in the brain by magnetic resonance imaging. Annals of Neurology. 1999; 45(2):265-9. http://dx.doi.org/10.1002/15318249(199902)45:2<265::AID-ANA21>3.0.CO;2-3

Narr KL, Thompson PM, Sharma T, Moussal J, Cannestra AF, Toga AW. Mapping morphology of the corpus callosum in schizophrenia. Cerebral cortex (New York, NY ,1991). 2000; 10(1):40-9. http://dx.doi.org/10.1093/ cercor/10.1.40

Narr KL, Cannon TD, Woods RP, Thompson PM, Kim S, Asunction D, van Erp TG, Poutanen VP, Huttunen M, Lönnqvist J, Standerksjöld-Nordenstam CG, Kaprio J, Mazziotta JC, Toga AW. Genetic Contributions to Altered Callosal Morphology in Schizophrenia. The Journal of Neuroscience. 2002; 22(9):3720-9. PMid:11978848.

O'Dwyer L, Lamberton F, Bokde ALW, Ewers M, Faluyi YO, Tanner C, Mazoyer B, O’Neill D, Bartley M, Collins DR, Coughlan T, Prvulovic D, Hampel H. Multiple indices of diffusion identifies white matter damage in mild cognitive impairment and Alzheimer's disease. PLoS one. 2011; 6(6):e21745. PMid:21738785 PMCid:PMC3128090. http://dx.doi.org/10.1371/journal. pone.0021745

Oh JS, Suk Park K, Chan Song I, Ju Kim S, Hwang J, Chung A, Kyoon Lyoo I. Fractional anisotropy-based divisions of midsagittal corpus callosum. Neuroreport. 2005; 16(4):31720. http://dx.doi.org/10.1097/00001756-200503150-00002

Park HJ, Kim JJ, Lee SK, Seok JH, Chun J, Kim DI, Lee JD. Corpus callosal connection mapping using cortical gray matter parcellation and DT-MRI. Human Brain Mapping. 2008; 29(5):503-16. PMid:17133394. http:// dx.doi.org/10.1002/hbm.20314

Park JS, Yoon U, Kwak KC, Seo SW, Kim SI, Na DL, Lee JM. The relationships between extent and microstructural properties of the midsagittal corpus callosum in human brain. NeuroImage. 2011; 56(1):174-84. PMid:21281715. http://dx.doi.org/10.1016/j.neuroimage.2011.01.065

Rajapakse JC, Giedd JN, Rumsey JM, Vaituzis AC, Hamburger SD, Rapoport JL. Regional MRI measurements of the corpus callosum: a methodological and developmental study. Brain and Development. 1996;18(5):379-88. http:// dx.doi.org/10.1016/0387-7604(96)00034-4

Rumsey JM, Casanova M, Mannheim GB, Patronas N, De Vaughn N, Hamburger SD, Aquino T. Corpus callosum morphology, as measured with MRI, in dyslexic men. Biological Psychiatry. 1996; 39(9):769-75. http://dx.doi. org/10.1016/0006-3223(95)00225-1

Rosas HD, Lee SY, Bender AC, Zaleta AK, Vangel M, Yu P, Fischl B, Pappu V, Onorato C, Cha JH, Salat DH, Hersch SM. Altered white matter microstructure in the corpus callosum in Huntington's disease: implications for cortical disconnection. NeuroImage. 2010; 49(4):29953004. PMid:19850138 PMCid:PMC3725957. http://dx.doi. org/10.1016/j.neuroimage.2009.10.015

Thompson PM, Narr KL, Blanton RE, Toga AW. Mapping structural alterations of the corpus callosum during brain development and degeneration. Proceedings of the NATO ASI on the corpus callosum. 2003; 93-130.

Von Plessen K, Lundervold A, Duta N, Heiervang E, Klauschen F, Smievoll AI, Ersland L, Hugdahl K. Less developed corpus callosum in dyslexic subjects-a structural MRI study. Neuropsychologia. 2002; 40(7):1035-44. http:// dx.doi.org/10.1016/S0028-3932(01)00143-9

Wahl M, Lauterbach-Soon B, Hattingen E, Jung P, Singer O, Volz S, Klein JC, Steinmetz H, Ziemann U. Human motor corpus callosum: topography, somatotopy, and link between microstructure and function. Journal of Neuroscience. 2007; 27(45):12132-8. PMid:17989279. http://dx.doi.org/10.1523/JNEUROSCI.2320-07.2007

Witelson SF. Hand and sex differences in the isthmus and genu of the human corpus callosum. A postmortem morphological study. Brain. 1989; 112 (Pt 3):799-835. PMid:2731030. http://dx.doi.org/10.1093/brain/112.3.799

Witelson SF, Goldsmith CH. The relationship of hand preference to anatomy of the corpus callosum in men. Brain Research. 1991; 545(1-2):175-82. http://dx.doi. org/10.1016/0006-8993(91)91284-8

\section{Authors}

Leticia Rittner*, Pedro Ferro Freitas, Roberto de Alencar Lotufo

School of Electrical and Computer Engineering, University of Campinas - UNICAMP, CEP 13083-852, Campinas, SP, Brazil.

\section{Simone Appenzeller}

Department of Medicine, Rheumatology Unit, University of Campinas - UNICAMP, Campinas, SP, Brazil. 